\title{
Tagungsbericht
}

\section{Viele erste Male - die GAL-Sektionentagung 2019 in Halle an der Saale}

https://doi.org/10.1515/iwp-2019-2050

Die im Turnus von zwei Jahren stattfindende Sektionentagung der Gesellschaft für angewandte Linguistik (GAL) wartete in diesem Jahr mit einem besonders vielfältigen und umfangreichen Tagungsprogramm in Halle an der Saale auf. Geleitet von der Abteilung für Sprechwissenschaft unter Prof. Ines Bose gab es neben Beiträgen zu fünfzehn Fachsektionen das neuartige Format des „Marktplatzes“ sowie einen Posterslam und die Verleihung des Nachwuchspreises zu erleben. Dieser Beitrag versucht sich in einem zusammenfassenden Überblick des Tagungsverlaufs.

This year, the Sectional Conference of the GAL, which takes place every two years, offered a particularly varied and extensive conference programme in Halle an der Saale. Led by the Department of Linguistics under Prof. Ines Bose, the participants inside could experience contributions to fifteen specialized sections, the new format of the "market place" as well as a poster slam and the award of the new generation price. This article tries to summarize the course of the conference.

Die GAL-Sektionentagung 2019 war mit 202 Vorträgen die bisher größte und längste Sektionentagung der Gesellschaft für Angewandte Linguistik. Sie fand vom 17. bis 20. September 2019 an der Martin-Luther-Universität Halle-Wittenberg statt und wurde von der Abteilung Sprechwissenschaft und Phonetik unter der Leitung von Prof. Ines Bose organisiert. 321 Tagungsgäste aus erstmals 17 Ländern trugen in 15 Sektionen mit Vorträgen, Postern und Gesprächen zu vielfältigen Diskussionen bei, die sich hier nicht annähernd abbilden lassen. Daher soll dieser Bericht einen ersten Einblick in das umfangreiche Pro-

\footnotetext{
*Kontaktperson: Stefanie Klein, Martin-Luther-Universität Halle-Wittenberg, Germanistisches Institut, Fachwissenschaft Deutsch an Grundschulen, Adam-Kuckhoff-Straße 15, 06099 Halle (Saale), E-Mail: stefanie.klein@germanistik.uni-halle.de Justine Schöne, Martin-Luther-Universität Halle-Wittenberg, Germanistisches Institut, Abteilung für Sprachwissenschaft, Luisenstraße 2, 06099 Halle (Saale), E-Mail: justine.herbst@germanistik.uni-halle.de
}

gramm ermöglichen. Für Interessierte lohnt sich zusätzlich ein Blick in das Book of Abstracts ${ }^{1}$, das die Kerngedanken der Vorträge zusammenfasst.

Gemeinsam mit der doctima GmbH verlieh die Gesellschaft für Angewandte Linguistik zunächst den Nachwuchspreis „Berufliche Kommunikation“ an Heidi Seifert von der Uni Hannover für ihre Dissertation. Da der Preis auch für Bachelor- und Masterarbeiten ausgeschrieben wird, die jedoch häufig unprämiert blieben, überraschte die Jury mit einer zweiten Verleihung: Alexa Wenisch von der Uni Wien wurde zusätzlich für ihre BA-Thesis ausgezeichnet. Im Anschluss an die Poster Session und den Poster Slam wurden dann die besten Nachwuchsprojekte prämiert. Auch das war neu: Bisher gab es eine Auszeichnung für ein Posterprojekt. Dieses Mal wurden Justine Schöne für ihr Poster und David Fujisawa für seinen Beitrag zum Poster Slam mit einem Preis bedacht.

Zum ersten Mal gab es zudem ein Plenum und einen Marktplatz zu digitalen Sprachressourcen für die Angewandte Linguistik. Der Marktplatz war gefüllt mit Informationsständen, an denen existierende Sprachressourcen und digitale Werkzeuge für die empirische linguistische Forschung vorgestellt wurden. Mit dabei waren Projekte wie MoCoDa2, „ein Forschungs- und Lehrkorpus zur Sprache und Interaktion in mobiler Messaging-Kommunikation" und weitere öffentlich zugängliche Datenbanken und Korpora. Nachfolgend werden die einzelnen Sektionen in aller Kürze - vorgestellt.

Fachkommunikation: Professionskommunikation als Sprache im Beruf: Die Komplexität beruflicher Kommunikation sowie die neuen Herausforderungen an Fachkommunikation standen im Mittelpunkt der ersten Sektion. Von der Frage darüber, wie man richtig manipuliert über die „Fallstricke“ der Fachkommunikation in der IT-Branche bis hin zu Handlungsstrukturen und Vertextungsstrategien in juristischen Stellenanzeigen ergab sich ein thematisch brei-

1 Dieses ist online verfügbar auf der Tagungswebsite oder direkt unter https:/gal-tagung-2019.sprechwiss.uni-halle.de/files/2019/08/ Book-of-Abstracts.pdf. 
tes Feld. Praktische Ziele, wie die Verbesserung der fachsprachlichen Kenntnisse für Migrantinnen und Migranten als Erleichterung in den Berufseinstieg oder die Optimierung von Texten im beruflichen Alltag wechselten sich ab mit Betrachtungen zu Stellenanzeigen, interkulturellem EMail-Verläufen oder der Frage nach dem Experten- und Laientum von wissenschaftlich Tätigen.

Gesprächsforschung: "How to get things done" - Instruktionen und Aufforderungen in der Interaktion: Wie genau kann eine zielgerichtete Instruktion/Aufforderung in Schule/Beruf/Freizeit bzw. Alltag aussehen? Wie können Handlungs- und Sprachmuster besser beschrieben und erforscht werden? Diese und viele weitere Fragen eröffneten sich. In den Feldern Schule \& Lernen wurden Themen wie Beratungsgespräche, Aufgabenstellungen im Bereich Deutsch als Zweitsprache, dem funktional einsprachigen Englischunterricht oder kooperativen Lernsettings angesprochen. Aber auch die Fahrschule als Ort permanenter Instruktionen wurde thematisiert. Hingegen zeigten sich in der Arbeitswelt Instruktionen im Kontext hierarchiefreier Kooperation unterschiedlicher Berufsgruppen von Interesse.

Textlinguistik \& Stilistik: Kleine Texte: Ab wann ist ein Text ein kleiner Text? Welche Merkmale kennzeichnen kleine Texte und wie werden diese in alläglichen kleinen Texten wiedergefunden? Wie vielfältig ist diese Textsorte? Die Sektion widmete sich dieses Mal ganz dieser kleinen und doch bemerkenswerten Textform. Ausgehend von einem theoretischen Beitrag zu sehr kleinen Texten und ihren Erscheinungsformen wurden zahlreiche Praxisbeispiele präsentiert. Dazu gehörten Ansichtskarten, Aushänge an öffentlichen Türen, T-Shirts von Jugendlichen, Social-Media-Texte oder auch Untertitel. Zudem wurde der kritische Umgang mit kurzen „pseudo-wissenschaftlichen" Texten sowie ein Gegenbeispiel, die sogenannten „Rapid Reactions“ und „Research in Context“, diskutiert.

\section{Medienkommunikation: Fankulturen und Fankom-} munikation: Viele Menschen bezeichnen sich als Fan von etwas oder jemandem. Doch ab wann ist ein Fan ein Fan? Was gehört zum „doing being a fan“ dazu und wie gestaltet sich die Beziehung zwischen Fan und Fanobjekt? Die Sektion eröffnete die vielfältigen Perspektiven von Fantum im medialen Kontext. Die Gestaltungsformen und Merkmale von „doing being a fan“ unterscheiden sich sowohl von Plattform zu Plattform (etwa bei Twitter, YouTube, in Foren oder bei Rezensionen) als auch zwischen Gruppen und Themen stark. Dabei zeigen sich bestimmte wiederkehrende Praktiken und auch öffentliche Diskurse, in denen diese ausgehandelt werden. Jedoch bezieht sich das Fantum nicht allein auf die Rezeption, sondern kann durch die Produktion eigener Videos oder Texte (Fanfiction) aktiv werden.

Mehrsprachigkeit: 25 Jahre Mehrsprachigkeit als Thema bei der GAL: Nach 25 Jahren der wissenschaftlichen Auseinandersetzung mit Mehrsprachigkeit lud die diesjährige Sektion sowohl zu einem historischen Rückblick als auch zu einer „Bestandsaufnahme“ und einem Blick in die Zukunft ein. Welche Themen sind aktuell, welche wurden bereits ausreichend beleuchtet, welche sollten aufgenommen werden? Das Spektrum reichte vom Blick auf den internationalen Vergleich über die deutsche Hochschulpolitik bis hin zu anwendungsbezogenen Aspekten. Dabei wurde sowohl Material zur mehrsprachigen Bildung als auch unterschiedliche Settings (etwa das Theaterspiel) oder die Auswirkungen der Bildungssituation in den Herkunftsländern von Geflüchteten betrachtet. Zugleich wurde die Einstellung zu einer Mehrsprachigkeit im Kontrast zur Monolingualität thematisiert. Aber auch methodische Fragen, wie $\mathrm{zu}$ Transkriptionen mehrsprachiger Unterrichtssituationen waren $\mathrm{zu}$ finden.

Sprachdidaktik: Empirische Forschung und sprachdidaktische Entwicklung: Welche Beiträge kann die empirische Forschung zur Entwicklung der Sprachdidaktik beitragen und welche Leistungen sind ihr bereits gelungen? In diesem Rahmen bewegten sich die Beiträge zur diesjährigen Sektion der Sprachdidaktik. Von Interesse waren hierbei mögliche Hürden der Sprach bzw. Textproduktion von Lernenden (wie etwa Wortschatz oder mangelnde Kenntnis über Konnektoren), differenzierendes Lesen und das Microscaffolding in Vorbereitungsklassen. Weiterhin konnten Ergebnisse zu der Fähigkeit von Schülerinnen und Schülern, die Akzeptabilität grammatischer Strukturen graduell zu bewerten, vorgestellt werden. Klar wurde vor allem: Digitale Produkte und Lernumgebungen können nur dann gewinnbringend eingesetzt werden, wenn sie als Medium/Methode an das Lernziel angepasst werden und nicht umgekehrt.

\section{Sprachkritik: Digitale Kommunikation und die Rolle} der Sprachkritik: Die menschliche Kommunikation verändert sich in zunehmendem Maße. Insbesondere in der digitalen Welt lassen sich auf pragmatischer Ebene vielfältige Entwicklungen ausmachen. Nicht nur die Art und Weise, wie geschrieben wird, sondern auch die Reaktionen darauf verändern sich. Die Beiträge der diesjährigen Sektion zu Sprachkritik bewegten sich daher in diesem Rahmen und nahmen das Kommunikationsverhalten im 
Netz in den Blick. Ausgehend von einem einführenden Beitrag in dieses Feld dienten unterschiedliche digitale Settings, wie etwa die Online-Hassrede, Onlineforen zu psychosozialen Themen, Social Media oder nicht-institutionelle Deutschlernvideos als beispielhafte Diskursobjekte. Hierbei zeigten sich sowohl wiederkehrende argumentative Muster als auch spezifische digitale Praktiken. Zentrale Stichworte scheinen etwa „Wertschätzung“, „Verrohung“ und die „Verschiebung des Sagbarkeitsfeldes“ zu sein.

Interkulturelle Kommunikation \& mehrsprachige Diskurse: Interkulturelle Kommunikation meets Angewandte Linguistik - oder: Schatz, wir müssen reden! Interkulturelle Kommunikation - eine (fast) ausgestorbene Forschungsrichtung?! Mit dieser Frage bzw. der Daseinsberechtigung des Begriffes selbst und den dahinterliegenden sprachlichen Phänomenen befasste sich die Sektion in diesem Jahr eingehend. Interkulturelle Kommunikation findet z.B. im Bereich Digitalisierung, in der Schulsozialarbeit, in MINT-Fächer-Communities, der Familie, interkulturellen Teams oder Social Media statt. Diese Räume eröffnen dementsprechend vielfältige Diskussions- und Erkenntnisrahmen, wie etwa die Frage nach einer interkulturellen Nutzung von Emojis, dem „doing being a familiy“ in zugewanderten Familien. Insgesamt zeigte sich, dass das Thema alles andere als ausgedient hat - es verändert sich lediglich entsprechend seiner äußeren Bedingungen.

Lexikographie \& Kollokationen: Varianz im Wörterbuch: Die Erhebung und Untersuchung von Varietäten ist eine etablierte Aufgabe der Sprachwissenschaft. Das stellt die Lexikographie jedoch vor eine Herausforderung: Wörterbücher können immer nur einen Teil der Sprache abbilden, aber nie ihre gesamte lexikalische Vielfalt. Wie gehen einzelne Wörterbücher mit Varianz um? Wie lässt sich der Varianz mehr Raum geben? Neben grundlegenden Überlegungen wurden auch konkrete Umsetzungen präsentiert, wenn es bspw. um rechtssemantische Varianz oder um Mundarten in Wörterbüchern ging.

Mediendidaktilk \& Medienkompetenz: Bildung in der digitalen Welt: Praxisbeispiele aus Linguistik, Sprachund Mediendidaktik: Ausgehend vom KMK-Strategiepapier fragte diese Sektion nach dem konkreten Einsatz digitaler Medien als Instrumente für die Gestaltung von Lehren und Lernen. Die vorgestellten Projekte und Anregungen deckten dabei die komplette Breite ab: Tools wie Padlet wurden zur Kollaboration genutzt, Studierende erstellten eigene Fragen in QuizzApps wie ArsNova, Peer-
Reviews verbesserten die Qualität von Studierendentexten. Auch Lern- bzw. Erklärvideos wurden vorgestellt - sowohl von Dozierenden für Studierende produzierte, interaktive Videos in einem größeren Setting als auch solche, die von Grundschulkindern durch Lernen durch Lehren hergestellt wurden.

Migrationslinguistik: Herkunftssprachlicher Unterricht: Im Round-Table wurde intensiv diskutiert, was herkunftssprachlicher Unterricht nutzt und wie er aussehen kann. Wie geht es den Schülerinnen und Schülern damit, mit ihren Herkunftssprachen in das Bildungssystem einbezogen zu werden? Gewinnen sie dadurch etwas? Können dadurch Bildungsrückstände aufgeholt werden? Nicht nur dort war diese Form des Unterrichts bestimmend für die Sektion: In einzelnen Vorträgen ging es um die Auseinandersetzung mit seiner Bedeutung aus Elternsicht und aus der der Vortragenden. Neben diesen grundsätzlichen Fragen wurden auch konkrete Projekte vorgestellt, die die schriftsprachlichen Fähigkeiten, den bilingualen Wortschatz oder aber konkrete Schreibaufgaben in den Fokus nahmen.

Phonetik \& Sprechwissenschaft: Transkription und Annotation gesprochener Sprache und multimodaler Interaktion: Konzepte, Probleme, Lösungen: Mittelpunkt der Sektion war die Transkriptions- und Annotationsarbeit. Insbesondere mit Blick auf den Einfluss technologischer Entwicklungen stand dabei vor allem die Annotation interaktiven Verhaltens im Zentrum. Eine Besonderheit bot das Kolloquium „Zur Transkription von Standardsprache in Aussprachedatenbanken: Konzepte, Probleme, Lösungen“ . Dort wurden Möglichkeiten und Grenzen der Transkription ausführlich u.a. im Hinblick auf die Zielgruppe der Deutschlernenden diskutiert.

Schreibwissenschaft: Schreibwissenschaft methodisch - gestern, heute, morgen: Diese neue Sektion stand völlig unter dem Aspekt der Prozessorientierung: Neben historischen Entwicklungen und dem aktuellen Stand war in mehreren Vorträgen, Postern und Workshops die Produktion von Texten und die Förderung von Kompetenzen von Interesse. Es ging u. a. um kooperative, journalistische und fremdsprachliche Textproduktion sowie allgemein um Mehrsprachigkeit. Überschneidungen mit didaktischen Feldern ließen sich dabei nicht nur bei den Fragen nach Schreibimpulsen, schriftlichen Feedbackkommentaren auf Studierendentexte und der Förderung akademischer Schreibkompetenz finden. 
Soziolinguistik: Soziolinguistik 2019: Theorien, Schlüsselkonzepte und Zugänge: Wie lässt sich eigentlich das Feld der Soziolinguistik bestimmen? Es vereint immerhin verschiedene Zugänge und Methoden und tangiert weitere Disziplinen wie die Diskurslinguistik. Ziel der Sektion war daher eine Bestandsaufnahme dieser pluralistischen Disziplin. So heterogen wie die Disziplin zeigten sich auch die Beiträge der Sektion: Grundsätzliche Fragen zur Selbst-Subjektivierung und der Rolle der Sprecherinnen und Sprecher über Methodenangebote wie der korpuslinguistischen Analyse sprachlicher Muster bis hin zu konkreten Phänomenen wie „präpositionslose Konstruktionen" wurden behandelt.

Übersetzungs- \& Dolmetschwissenschaft: Altes bewahren, Neues wagen - Neue Wege für die Translationswissenschaft? Die „neuen Wege“ der Translationswissenschaft bieten zu einem großen Teil Technologien zur Unterstützung von Translationsprozessen. Die maschinelle Übersetzung trägt $\mathrm{zu}$ einem effizienten Übersetzungsprozess bei, doch zeigen sich darin auch Probleme: So wurden bei der Sektion u. a. Textsorten diskutiert, die sich nicht einfach maschinell übersetzen lassen. Es stünden bspw. Werbetexte immer in einem kulturellen Kontext, der bei jeder Übersetzung berücksichtigt werden müsse. Auch die Frage, wie sich Leichte Sprache übersetzen lässt, stand im Fokus der Sektion. Neben den Chancen und Anforderungen maschineller Übersetzungen wurden aber auch die Wechselwirkungen zwischen den diversen Bereichen der Translationswissenschaft und -praxis untereinander und zu anderen Disziplinen diskutiert. Sie böten neue Anforderungen an Wissenschaft und Praxis, denen sich die Translationswissenschaft stellen müsse.

\section{Weiterführende Links und Informationen}

Informationen zur Sektionentagung:

https://gal-tagung-2019.sprechwiss.uni-halle.de/

Programm:

https://www.conftool.org/gal2019/sessions.php

Einladungsheft (Calls der Sektionen):

https://gal-tagung-2019.sprechwiss.uni-halle.de/files/

2019/04/GAL2019-Einladungsheft.pdf

Abstracts: https://gal-tagung-2019.sprechwiss.uni-halle. de/files/2019/08/Book-of-Abstracts.pdf

Informationen zur Poster-Session:

https://gal-tagung-2019.sprechwiss.uni-halle.de/

programm/poster-session/
Deskriptoren: Tagung, GAL 2019, Sprachwissenschaft, Digitalisierung, Forschung, Projekt, Martin-Luther-Universität Halle-Wittenberg



\section{Stefanie Klein}

Martin-Luther-Universität

Halle-Wittenberg

Germanistisches Institut, Fachwissenschaft

Deutsch an Grundschulen

Adam-Kuckhoff-Straße 15

06099 Halle (Saale)

stefanie.klein@germanistik.uni-halle.de

Stefanie Klein ist wissenschaftliche Mitarbeiterin und Doktorandin in der Abteilung Fachwissenschaft Deutsch für das Lehramt an Grundschulen im Germanistischen Institut an der Martin-Luther-Universität Halle-Wittenberg. Ihr Forschungsschwerpunkt liegt auf dem universitären Einsatz von Erklärvideos in der germanistischen Sprachwissenschaft und dessen Evaluation.

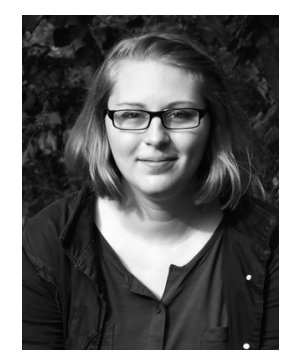

Justine Schöne

Martin-Luther-Universität Halle-Wittenberg

Germanistisches Institut, Abteilung für

Sprachwissenschaft

Luisenstraße 2

06099 Halle (Saale)

justine.herbst@germanistik.uni-halle.de

Justine Schöne ist wissenschaftliche Mitarbeiterin und Doktorandin in der Abteilung für Sprachwissenschaft des Instituts für Germanistik an der Martin-Luther-Universität Halle-Wittenberg. Sie forscht dort zu schulischen Erklärvideos als Bildungsmedium und multimodale Textsorten und legt den Fokus auf das multimodale Erklären und die Vermittlung von Wissen in den Videos innerhalb ihres Textsortennetzes. 